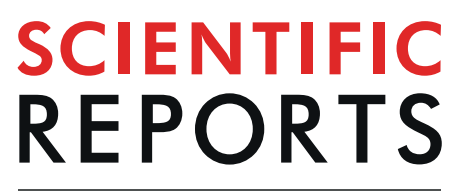

natureresearch

\title{
Intestinal Neurod1 expression impairs paneth cell differentiation and promotes enteroendocrine lineage specification
}

Hui Joyce Li ${ }^{1}$, Subir K. Ray ${ }^{1}$, Ning Pan ${ }^{2,4}$, Jody Haigh ${ }^{3}$, Bernd Fritzsch $\mathbb{1}^{2}$ \& Andrew B. Leiter ${ }^{1 *}$

Transcription factor Neurod 1 is required for enteroendocrine progenitor differentiation and maturation. Several earlier studies indicated that ectopic expression of Neurod1 converted non- neuronal cells into neurons. However, the functional consequence of ectopic Neurod1 expression has not been examined in the GI tract, and it is not known whether Neurod1 can similarly switch cell fates in the intestine. We generated a mouse line that would enable us to conditionally express Neurod 1 in intestinal epithelial cells at different stages of differentiation. Forced expression of Neurod1 throughout intestinal epithelium increased the number of EECs as well as the expression of EE specific transcription factors and hormones. Furthermore, we observed a substantial reduction of Paneth cell marker expression, although the expressions of enterocyte-, tuft- and goblet-cell specific markers are largely not affected. Our earlier study indicated that Neurog3 + progenitor cells give rise to not only EECs but also Goblet and Paneth cells. Here we show that the conditional expression of Neurod1 restricts Neurog3+ progenitors to adopt Paneth cell fate, and promotes more pronounced EE cell differentiation, while such effects are not seen in more differentiated Neurod1 + cells. Together, our data suggest that forced expression of Neurod1 programs intestinal epithelial cells more towards an EE cell fate at the expense of the Paneth cell lineage and the effect ceases as cells mature to EE cells.

Intestinal epithelial cells are divided into two main categories - absorptive cells and secretory cells. The absorptive cells include enterocytes and the secretory cells include enteroendocrine (EE), goblet, Paneth, and tuft cells $s^{1,2}$. All five cell types are derived from common multipotent intestinal stem cells. The expression of the atonal basic helix-loop-helix (bHLH) transcription factor 1 (Atoh1) initiates the differentiation of progenitor cells that are subsequently either fated to EE cell lineages by the downstream transcription factor, Neurogenin-3 (Neurog3, Ngn3), or to Goblet and Paneth cell lineages upon expression of the transcriptional repressor, growth factor independent- $1(G \text { fil })^{3-7}$. The differentiation of Tuft cells, on the other hand, adopts an Atohl-independent pathway involving high expression of Sox $4^{8,9}$. EE cells are hormone-producing cells scattered individually throughout the mammalian gastrointestinal tract. These cells comprise less than $2 \%$ of the total cell population. The maturation and subtype specification of EE cells along crypt-villus axis require subsequent expression of a number of pro-endocrine transcription factors including Neurod1, Lmx1a, Nkx2-2, Insm 1, Arx, Isl1 and Pax4/6 $6^{10-19}$.

The expression of Neurod1, a member of the bHLH family of transcription factors, is restricted to pancreatic islets, intestinal endocrine, stomach and neuronal cells. It regulates the expression of the insulin gene in pancreatic beta cells $s^{20,21}$, the secretin gene in small intestine $e^{22}$, and is capable to reprogram fibroblasts to a neuronal fate ${ }^{23-25}$ or Schwann cells into neurons ${ }^{26}$. In addition, Neurod1 (Nd1) also functions in governing cell cycle exit in photoreceptor lineage ${ }^{27}$ and can cause cell cycle arrest in in vitro cell culture ${ }^{28,29}$. Studies of Neurod 1 targeted deletion mutants revealed its important functions in development and maintenance in several developing systems including the central nervous system ${ }^{30-33}$, the peripheral nervous systems (including the inner ear) ${ }^{34-37}$, the EE cells of the GI tract ${ }^{7,38,39}$, as well as the beta cells of the pancreas ${ }^{21,40}$. Neurod 1 mutations have been found to cause

${ }^{1}$ Division of Gastroenterology, Department of Medicine, University of Massachusetts Medical School, 364 Plantation Street, Worcester, MA, 01605, USA. '2Department of Biology, University of lowa, lowa City, IA, 52242, USA. ${ }^{3}$ Department of Biomedical, Molecular Biology, Ghent University, Ghent, Belgium. ${ }^{4}$ Decibel Pharmaceutical, Boston, MA, USA. *email: andrew.leiter@umassmed.edu 
maturity-onset diabetes of the young and late-onset diabetes (Online Mendelian Inheritance in Man 606394) ${ }^{41}$. Recently, Neurod1 null mutations have been linked to ophthalmological phenotypes in humans ${ }^{42}$.

Neurod 1 is a direct transcriptional target of Neurog3 in intestinal EECs ${ }^{43,44}$. Early studies have shown that Neurog3 is sufficient to induce an endocrine program when expressed in the intestine of transgenic mice ${ }^{6}$. Recent single-cell RNA profiling of individual intestinal epithelial cells categorized both Neurog3 and Neurod 1 as markers for immature ${ }^{45}$ or differentiating ${ }^{46}$ EEC precursors, suggesting a function of Neurod 1 in early EEC differentiation. Another function of Neurod 1 is its ability to reprogram other cell types into neurons through altering chromatin and transcription factor landscapes ${ }^{47}$. These results prompted us to investigate if expression of Neurod 1 prior to Neurog 3 can override the default function of Neurog3 in EE cell differentiation. To exam-

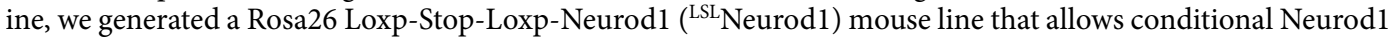
expression in Villin + cells (prior to Neurog3) by crossing with Vil-cre mice or Neurog3 + cells (concomitant with Neurog3) by crossing with Neurog3-cre mice. In this manuscript, we provide data demonstrating that conditional gain-of-function of Neurod 1 prior to Neurog3 expression robustly increases EE cell numbers and this differentiation process is limited to a subset of the gut progenitor cells that are competent to adopt an endocrine fate.

\section{Results}

Conditional expression of Neurod1 in the intestinal epithelial cells. To test if Neurod1 can switch cell fate and promote EEC differentiation in the developing intestinal epithelium, we generated a conditional gain-of-function Rosa26-loxp-Stop-loxp(LSL) Neurod1-IRES-eGFP mice line. A 1,280 bp full-length mouse Neurod1 cDNA was cloned into the pEntry vector (Gateway ${ }^{\circledR}$, Life Science). The conditional Rosa $26^{\text {SL }}$ Neurod1 targeting vector was created through recombination of the pROSA26-DV1 destination vector with pENTR-mNeurod1 and targeted to the Rosa26 locus by electroporation into JM8F6 (C57BL/6) mouse embryonic stem (ES) cells ${ }^{48}$. We identified 7 out of 36 correctly targeted ES clones by PCR analysis using primers that cover both Rosa26 genomic sequence and the inserted transgenes (Fig. 1A). Clone A1 was chosen for C57BL/ 6 blastocyst injection to generate

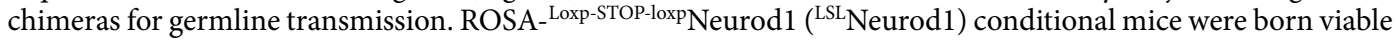
and fertile.

To determine whether ${ }^{\text {LSL }}$ Neurod 1 mice can be used to induce Neurod 1 expression by Cre-mediated recom-

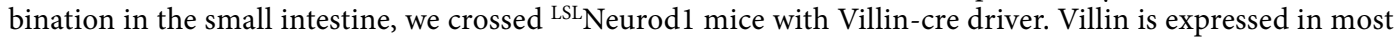
intestinal epithelial cells beginning at E13.5 ${ }^{49,50}$. Villin-cre-mediated excision of the Loxp-STOP-Loxp sequence allowed transcriptional read-through between the two LoxP sites in the Neurod1-IRES-eGFP bi-cistronic transgene, resulting in Neurod1 and EGFP expression. The expression of Neurod1 and EGFP transgenes were determined by qPCR for transcripts, and immunofluorescent microscopy and immunoblotting for Neurod 1 protein.

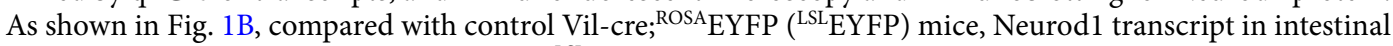
epithelial cells increased 4.4 -fold in Vil-cre, ${ }^{\text {LL }}$ Neurod 1 mice whereas EGFP expression level is much lower than

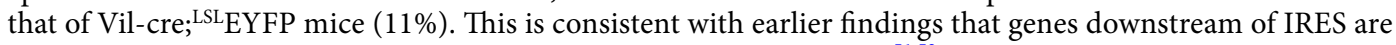
expressed at lower levels compared with the genes upstream of the IRES ${ }^{51,52}$.

The Neurod 1 and EG/YFP proteins in the intestine were detected by co-immunostaining with Neurod 1 antibody and EGFP antibody which recognized both EGFP and EYFP proteins on duodenal tissues from

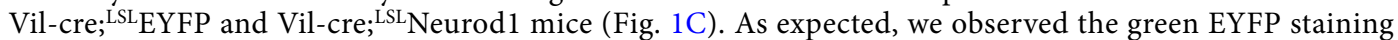
throughout crypt-villus structure of the intestine and sporadic Neurod1 nuclei staining (red arrow) in control

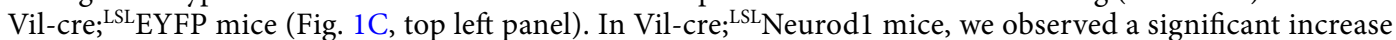
in nuclear Neurod1-positive cells along the intestinal epithelium, mostly in the crypts from the duodenum $(39 \% \pm 11 \%)$ to the colon $(32 \% \pm 3 \%)$ (Fig. 1D). Approximately $10 \%$ of the Neurod1-positive cells appeared as epithelial ribbons in the crypt (Fig. 1C, bottom right panel). EGFP expression can be detected in both crypt and villi of those cells co-stained with Neurod1. We also analyzed proteins in whole cell lysates from Vilcre, ${ }^{\mathrm{LSL}} \mathrm{Neurod} 1$ mouse intestine that are recognized by the Neurod1 antibody in a Western blotting analysis (Fig. 1E). The antibody detected a single protein band (molecular weight of $50 \mathrm{kDa}$, the size of Neurod1 protein) with little or no background, further consolidating the above Neurod1 immunostaining results (Supplemental Figure).

Neurod1 expression in Villin + cells profoundly increases EE cell numbers. To determine if the

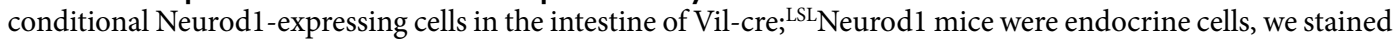
intestinal sections with a pan-endocrine marker Chromogranin A (ChgA $)^{53}$. A substantial increase in the number of ChgA + cells in both the duodenal $(11.1 \times)$ and colonic $(11 \times)$ epithelium were observed, compared to

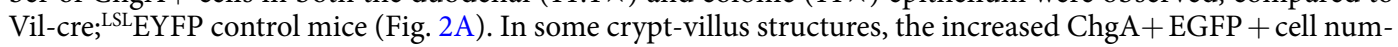
ber was more pronounced, with nearly all cells in the epithelium expressing ChgA. The increased EE cell population in Vil-cre; ${ }^{\mathrm{LSL}}$ Neurod 1 mice suggested that Neurod1 is able to direct EEC differentiation when ectopically expressed. Contrary to sporadic appearance of EECs in the normal intestine, we observed ChgA+EECs resided adjacent to each other (Fig. 2A,bottom right) in the intestines of Vil-cre; ${ }^{\mathrm{LSL}}$ Neurod1 mice, suggesting the loss of lateral inhibition function of Notch signaling by Neurod1, possibly through inhibiting Hes 1 function ${ }^{54}$.

We next determined if Neurod 1 increased the expression of genes associated with enteroendocrine function and differentiation by qPCR analysis. As shown in Fig. 2B, Neurod1 substantially increased the expression of many EEC hormones or associated genes including secretin $(S c t)$, cholecystokinin $(C c k)$, somatostatin $(S s t)$, tryptophan hydroxylase 1 (Tph1), gastric inhibitory polypeptide (Gip), and ghrelin; whereas glucagon transcripts did not show much change. The expression of a number of transcription factors associated with endocrine differentiation in the GI tract, including Nkx2-2, Neurog3, Lmx1a, Insm1, Isl1 and Arx were found considerably increased along with Neurod1 (Fig. 2C), consistent with the increase of EEC numbers.

We examined the effects of Neurod 1 on the expression of intestinal lineage specific genes including goblet cell (Muc2, Tff3, Klf4), enterocytes (ApoA4, Alpi, Fabp), Paneth cell (Lyz1, Dpp4) and Tuft cell (Dclk1, Siglec1) genes. qPCR assay shown in Fig. 3 revealed that the expression of Neurod 1 in Villin + cells had minimal effect on 
A.

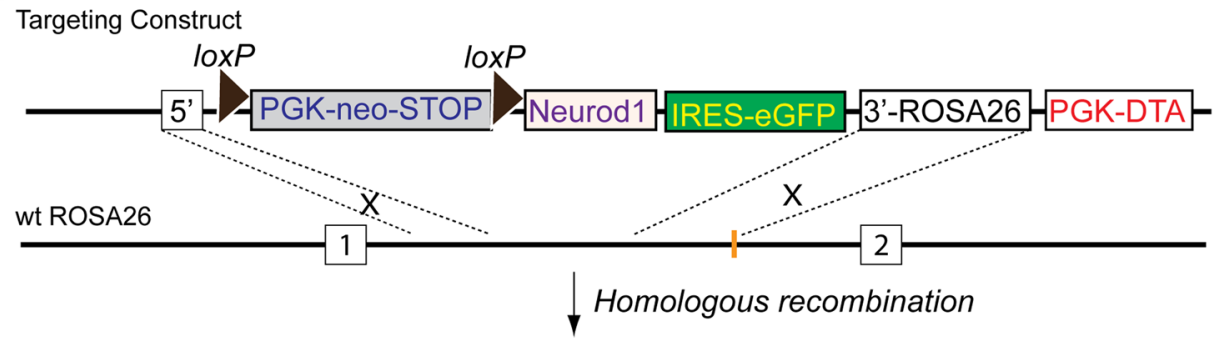

Knock-in allele
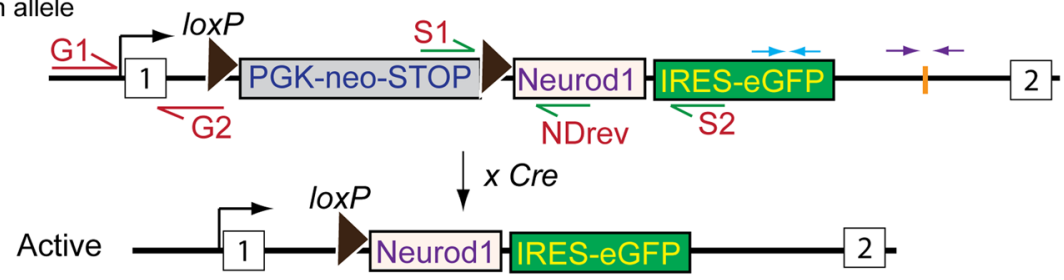

$\longrightarrow$ NeuroD1 + eGFP

B.

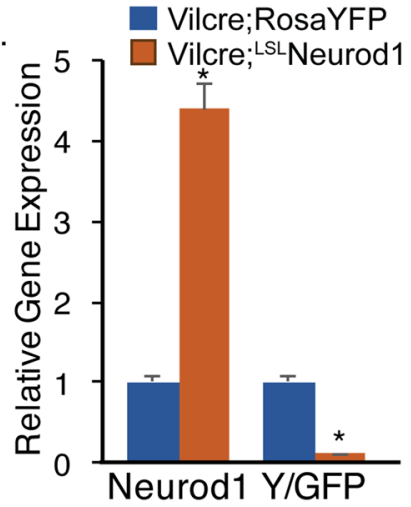

D.

\begin{tabular}{|l|c|c|c|}
\hline & Duod/Jj & lleum & Colon \\
\hline$\%$ Neurod1 + & $39 \pm 11 \%$ & $30 \pm 10 \%$ & $32 \pm 3 \%$ \\
\hline \multicolumn{4}{|c|}{ Paired p value $>0.3$}
\end{tabular}

E.

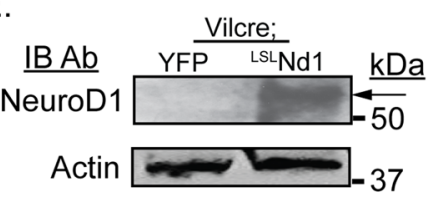

C.

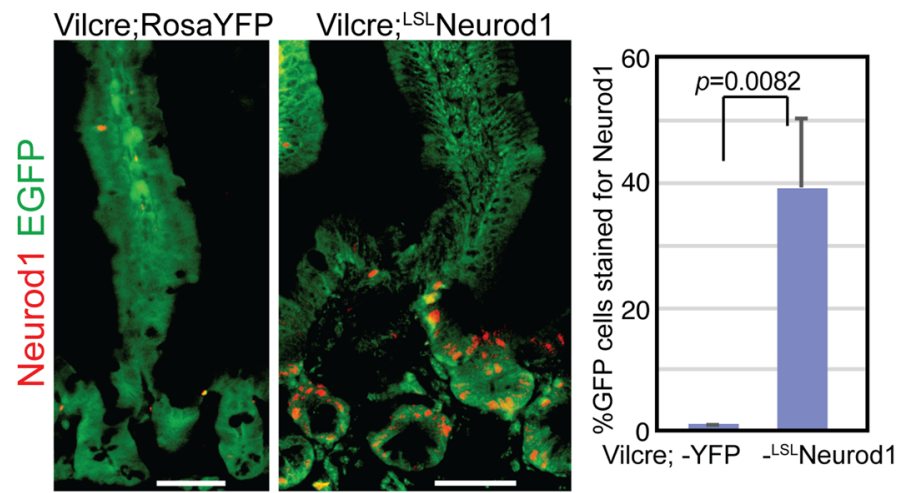

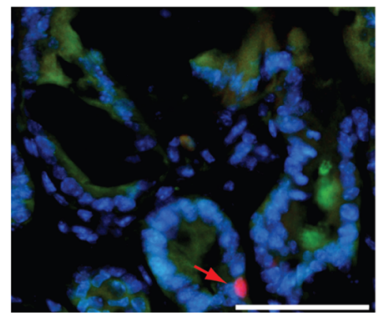

Vilcre;RosaYFP

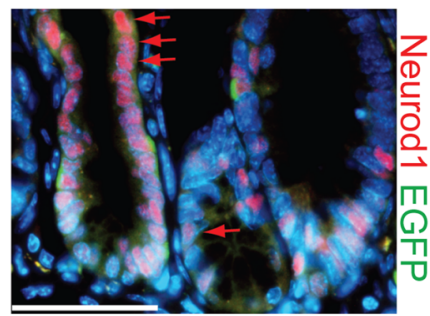

Vilcre; ${ }^{\text {LSLNNeurod1 }}$

Figure 1. Generation of a conditional Neurod1 mouse line at the ROSA26 locus. (A) In ROSA26 loci, the insertion of a loxP-PGK-neo-3xpA (STOP)-loxP sequence upstream of a knock-in Neurod1-IRES-eGFP gene prevents Neurod 1 from being transcribed. Cre-mediated deletion of the STOP sequence upon crossing with a driver-mice (e.g, Villin-Cre mice), allows the ROSA26 promoter to drive expression of the Neurod1-IRES-eGFP bi-cistronic fusion transcript. PCR primers used for verification of the indicated locations of recombined clone are shown by arrows of different colors, e.g. red arrows (G1:G2 primers); green arrows (S1:S2 and S1:NDrev); and blue and purple arrows. (B) Expression of Neurod1 message is relatively high in Vil-cre; ${ }^{\mathrm{LSL} N D}$ mice. Relative mRNA levels of Neurod1, EYFP or EGFP (normalized to $\beta$-actin) from indicated cells were shown. Values are mean $\pm \operatorname{SD}(n=3) ; * P \leq 0.0001$. (C) Representative double immunostaining for EYFP/EGFP (green) and Neurod1 (red) of duodenum tissues from Vil-cre; ROSA-YFP and Vil-cre; ${ }^{\mathrm{LSL}}$ Neurod1 mice. Bar graph shown summarized the $\% \mathrm{GFP}+$ cells stained for Neurod 1 from indicated mouse intestine. $p=0.0082$ as indicated. Scale bars $=50 \mu \mathrm{m}$. Red arrows pointed to nuclear Neurod1 staining. (D) Table summarized the \% Neurod $1+$ cells along intestinal tract. \pm represent standard deviation from at least three experimental replicates. The $\mathrm{p}$ values between each pair are: 0.45 (Duod to Colon), 0.72 (Ileum to Colon) and 0.33 (Duod to Ileum). These $p$ values are $>0.05$, indicating no significant difference among the three testing groups. (E) Expression of Neurod1 protein in Vil-cre; ${ }^{\mathrm{LSL}} \mathrm{Nd} 1 \mathrm{mice}$. Equal amounts of total protein from cell lysates of the duodenum of Vil-cre;YFP and Vil-cre; ${ }^{\mathrm{LSL}}$ Neurod 1 mice (top panel: $60 \mathrm{ug}$ protein/lane; bottom panel: 20 ug protein/lane) were separated on SDS-PAGE followed by immune-blotting with Neurod1 antibody (top panel, black arrow) or Actin antibody (bottom panel). 

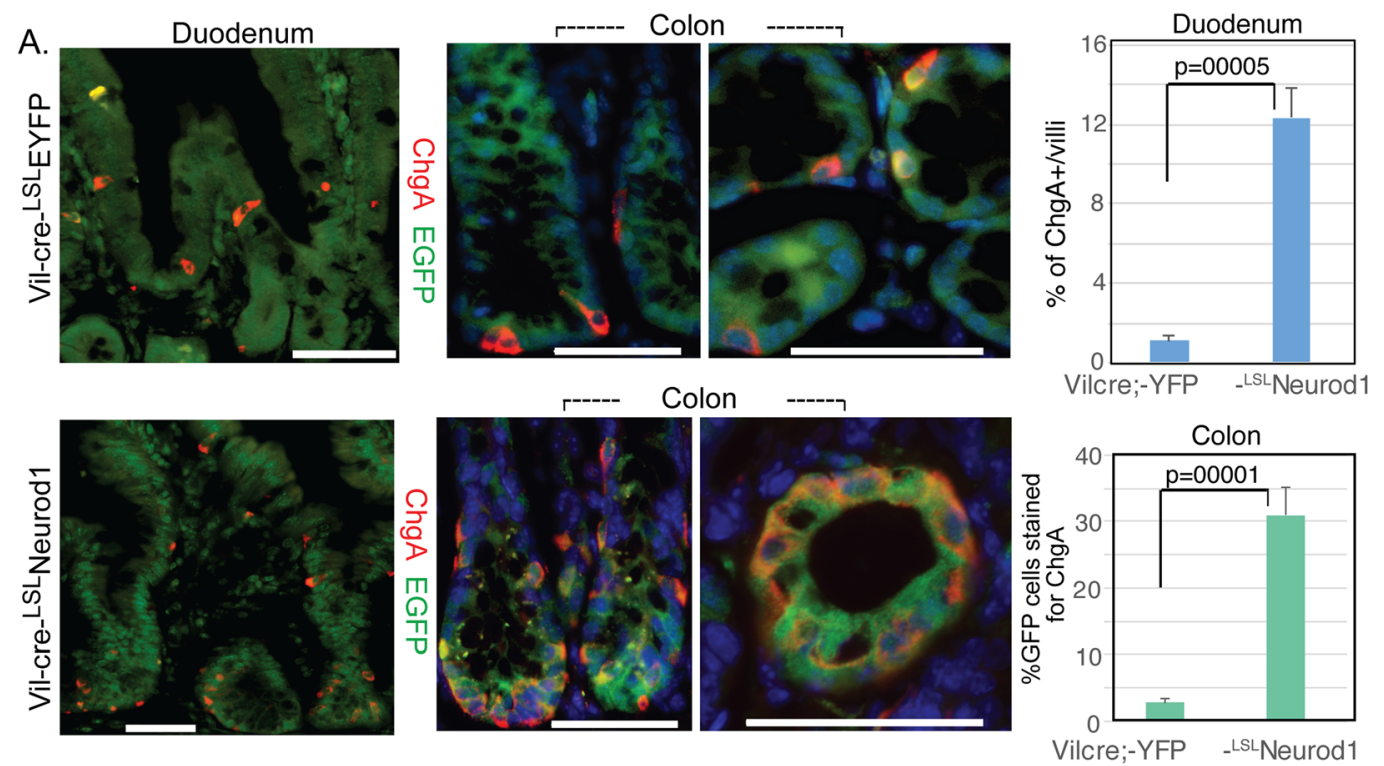

B.
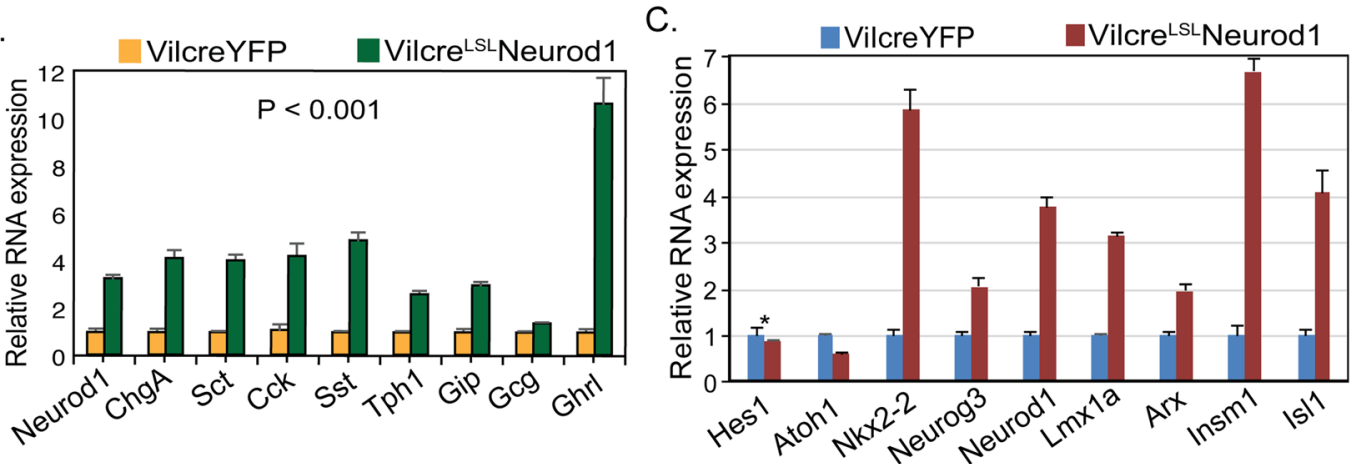

Figure 2. Expansion of EECs with conditional Neurod1 expression in Villin ${ }^{+}$cells. (A) Representative double immunostaining for EYFP/EGFP (green) and ChgA (red) of Crypt and Villus compartments from duodenum and colon samples of Vil-cre; ${ }^{\mathrm{LSL}} \mathrm{EYFP}$ and Vil-cre; ${ }^{\mathrm{LSL}} \mathrm{Neurod} 1$ mice. Bar graph on the right summarized the \% of ChgA + cells in the intestines between two mouse strains as indicated. The p values shown in the graph were: 0.0005 for Duodenum and 0.0001 for Colon. Nuclei were stained with Dapi. Scale bars $=50 \mu$ M. (B,C) Neurod1 in Villin + cells increased the expression of gastrointestinal hormones (B) and EEC-specific transcription factors (C). Bar graph represents normalized (normalized to $\beta$-actin) mRNA levels of the indicated genes. Values are mean $\pm \mathrm{SD}(\mathrm{n}=3)$. In $\mathrm{B}, P$ value of all hormone pairs $\leq 0.001$; in $\mathrm{C}, * P=0.21$, the rest $P \leq 0.001$.

expressions of genes specific for enterocytes, goblet and Tuft cells. However, expression of the Paneth cell specific maker genes $L y z 1$ and Dpp4 were reduced by approximately $60 \%$ and $50 \%$ respectively. These results suggested that upon Neurod1 expression in Paneth cell progenitors, their maturation is either blocked or shunt towards EEC lineage, possibly via a well-known negative feedback loop of Neurod1 on controlling Atoh1 expression level in developing system ${ }^{55,56}$.

However, not all Neurod 1 expressing cells were ChgA + (Fig. 4A, green arrow). We observed a $54.4 \% \pm 8 \%$ of

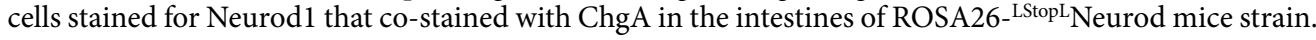

Neurod1 induction of the endocrine lineage depends on the differentiation stage. All five cell types in adult intestinal epithelium are constantly regenerated from multipotential ISCs. The expression of Neurog3 represents one of the earliest stages in the initiation of EEC specification whereas the subsequent expression of its target, Neurod1, occurs at a later stage of EEC differentiation when cells are restricted to an EEC cell fate $^{7}$. To determine the effect of Neurod 1 on cell fates in Neurog3 + early EEC progenitors or Neurod1+ late EEC

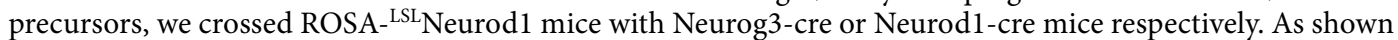
in Fig. 4, when Neurod1 expression was activated in Neurog3+ progenitor cells by the Neurog3-cre driver, we observed a small increase ( 2 fold) in ChgA + cell numbers. However, ectopic Neurod 1 expression in Neurod $1+$ cells resulted in a 58\% reduction of ChgA + EEC numbers (Fig. 4B). This suggested a potential function of Neurod 1 in promoting EEC differentiation and that may depend on the competency of the target progenitor cells.

Neurod1 expression in Neurog3 cells blocks their fate towards paneth cells. We further ana-

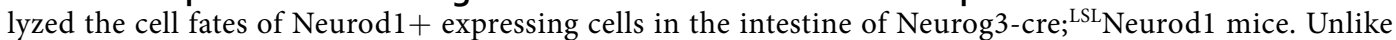
Neurog3 + cells in Neurog3cre;ROSA-EYFP mice that give rise to all three types of secretory cells, including 


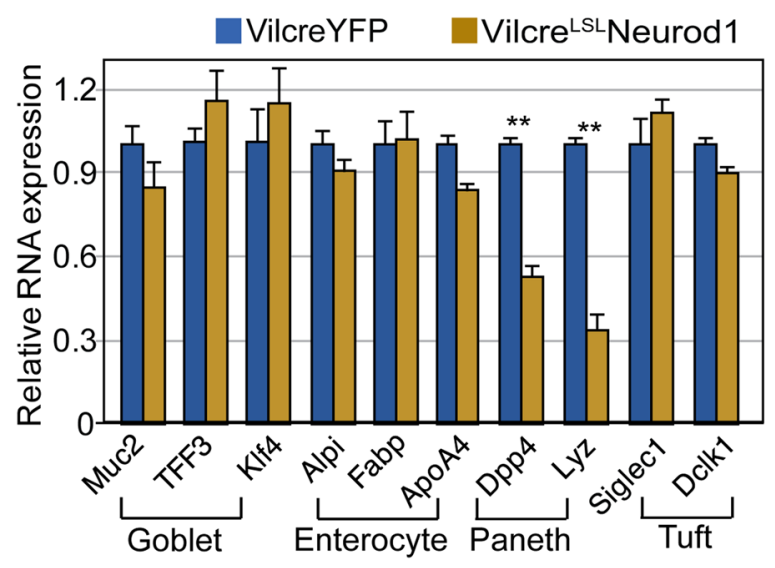

Figure 3. The effects of Neurod 1 expression in Villin + cells on the differentiation of non-EEC cell lineages. Neurod1 in Villin + cells did not affect marker gene expression for goblet cells and enterocytes. A reduction of Paneth cell marker expression was observed. Bar graph represents normalized (normalized to $\beta$-actin) mRNA levels of the indicated genes. Values are mean $\pm \mathrm{SD}(\mathrm{n}=3), * * P \leq 0.0001$; No substantial differences were seen of exogenous Neurod 1 expression on other lineage marker expression including enterocyte, goblet and tuft cells $(P \geq 0.05)$.

Paneth cells $(\sim 30 \%)$, goblet cells $(\sim 25 \%)$, and EEC cells $(\sim 40 \%)^{57}$, the Neurod 1 expressing Neurog $3^{+}$cells in

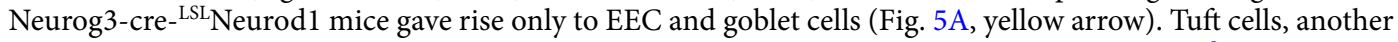
secretory cell type present in the small intestine, differentiate from an Atoh1-independent origin ${ }^{8}$. We observed an increase of ChgA + EEC cell numbers $\left(77 \%\right.$ for Neurog 3 cre $;{ }^{\mathrm{LSL}} \mathrm{Neurod} 1$ and $36 \%$ for Neurog $3 \mathrm{cre}^{\mathrm{ERT} 2}$;

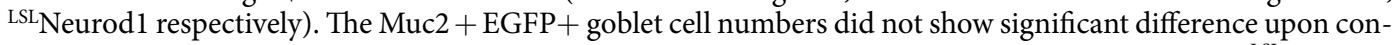
ditional Neurod 1 expression in Neurog3 progenitor cells $\left(p=0.25\right.$, Fig. 5A). We also crossed ROSA- ${ }^{\text {LSLNeurod } 1}$ mice with the Neurog3-cre-ER ${ }^{\mathrm{T} 2}$ inducible cre driver line to temporally induce Neurod 1 expression in Neurog3+ cells in adult mice. We observed a $35.5 \%$ increase in ChgA + cells $(\mathrm{p}=0.025)$, whereas a significant reduction of goblet cell number was seen $(\mathrm{p}=0.002$, Fig. $5 \mathrm{~B}$ ). The discrepancy of Neurod 1 effect on goblet cell differentiation may depend on the timing of Neurod 1 expression.

However, we did not observe EGFP-expression in Lyz ${ }^{+}$Paneth cells (Fig. 5A). Consistent with what we

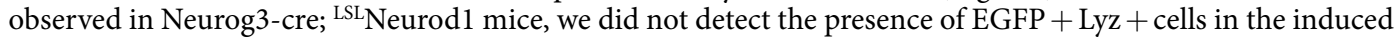
adult intestines (Fig. 5B). These data suggested that conditional Neurod 1 expression in Neurog $3+$ secretory progenitors blocked their differentiation to Paneth cells in both developing and adult mice.

\section{Discussion}

Here we generated a new Neurod 1 gain-of-function mouse line that enables conditional expression of Neurod 1 in Cre expressing cells at different stages of EEC maturation. Whether the intestinal cells would respond to ectopic Neurod 1 expression has not been examined. We reported here a substantial increase in the EEC numbers when Neurod 1 was conditionally expressed in Villin-Cre+ intestinal cells. Expression of Neurod 1 in Neurog3-Cre + cells only led to a modest increase in EEC numbers whereas reduction in EEC numbers was seen in Neurod1-Cre+ cells. As with the recent findings of Neurod 1 as a pioneer factor in the nervous system, our data suggest Neurod1 may function similarly in EEC differentiation and the effects are dependent on differentiation stage.

Sequential expression of bHLH transcription factor Neurogenin 3 and its target Neurod 1 is a hallmark of enteroendocrine (EE) lineage specification and maturation. We first considered Neurog $3+$ EEC progenitors since Neurog3 + cells are multipotent early EEC progenitor cells ${ }^{46,57}$ that give rise to EEC, Goblet and Paneth cells, although majority of Goblet and Paneth cells arise from Gfi+ secretory progenitors. We observed that the expression of Neurod 1 resulted in only $\sim 2$-fold increase of EEC numbers compared to WT, with no Neurod1 or ChgA epithelial ribbon staining. The increased EEC numbers may be from those Neurog3+ cells that fate to Paneth cells. Our data also suggested that EEC and Paneth cells arise at least in part from common progenitors, in line with recent lineage tracing experiments ${ }^{46}$.

Early lineage tracing in Villin-cre/ROSA26EGFP mice detected wide expression of EGFP throughout the whole intestinal epithelium, including all epithelial cells from intestinal stem cells at the crypt base and all types of matured epithelial cells. However, in the intestine of Villin-cre/ ${ }^{\text {LSL }}$ Neurod 1 mice, approximately $30 \%$ to $39 \%$ of epithelial cells stained for Neurod1 (Fig. 1D), mostly detected in cells located in the crypt and lower villus (Fig. 1C). This may be due to the shorter half-life of Neurod1 leading to decreased Neurod1 along crypt-villus structure. The sensitivity of Neurod1 antibody used in Immunostaining may not be able to detect low levels of Neurod1. Neurod1+ ribbon structure can only be seen in $\sim 10 \%$ of the whole intestine. A number of factors may contribute to such a discrepancy on the expression of transgenes between Vil-cre/L-Stop-L Neurod1 and Vil-cre/ROSA26R. Recent studies have shown that floxed alleles have different sensitivities to Cre-mediated recombination ${ }^{58}$. The recombination efficiency between different reporter alleles varies greatly on the nucleotide sequences flanking the LoxP sites, distance between loxP sites, the chromosomal location of floxed alleles and the levels of Cre activities. 

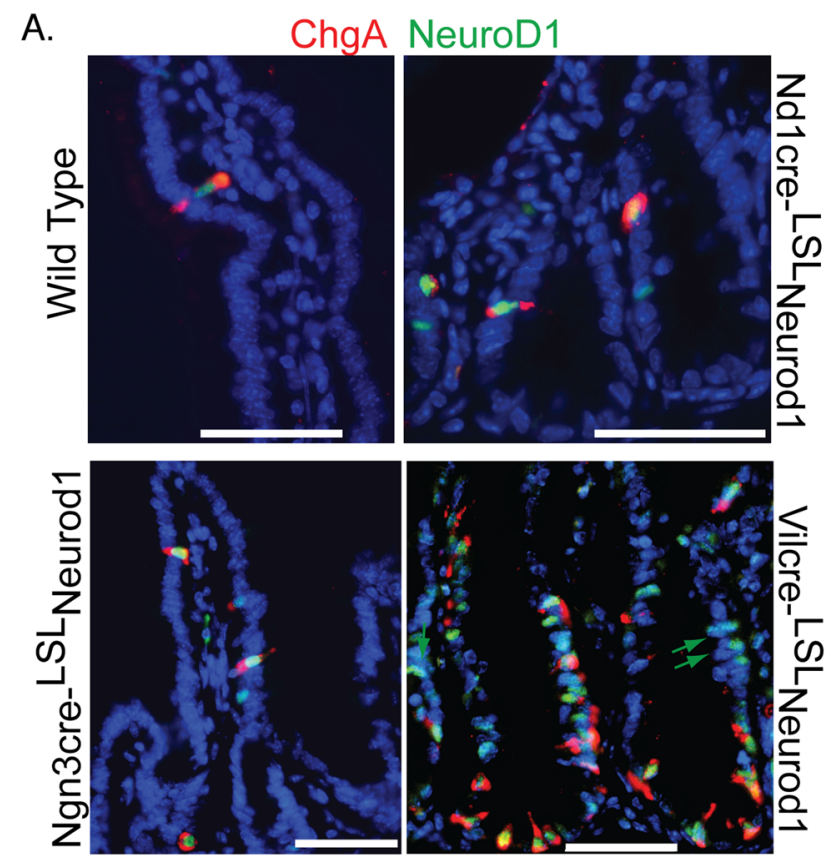

B.

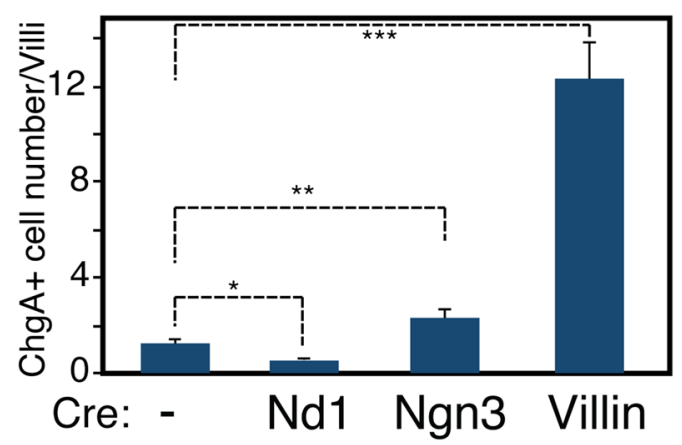

Figure 4. Conditional Neurod 1 expression in different EEC differentiation stages. Targeted Neurod1 expression in Neurod1+, Neurog3+ and Villin + cells were driven by Neurod1-cre, Neurog3-cre and Vil-cre mice respectively. (A) Duodenum sections from WT, Neurod1-cre ${ }^{\mathrm{LSL}}$ Neurod1, Neurog3-cre ${ }^{\mathrm{LSL}}$ Neurod1 and Vil-cre ${ }^{\mathrm{LSL}}$ Neurod1 were stained with antibodies against ChgA (red) and Neurod1(green). DAPI (blue) stains for nuclei. Scale bar $=50 \mu \mathrm{M}$. (B) The number of ChgA ${ }^{+}$cells in the intestines of indicated Cre- ${ }^{\text {LSL }}$ Neurod 1 mice were quantified as shown in the bar graph. Error bars represent standard deviation from at least three experimental replicates. $* P=0.007 ; * * P=0.004, * * * P \leq 0.0001$.

Unlike normal EECs present as individual mucosal endocrine cells, the ChgA + epithelial ribbon structure

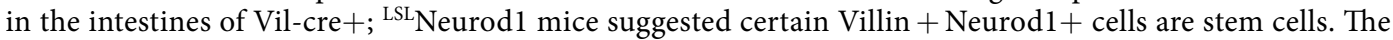
Intestinal cell types including EECs arise from intestinal stem cells (ISCs) located at the crypt base. The existence of two stem cell pools in the intestinal crypt is well recognized: actively Lgr5 + cycling columnar basal cells (CBCs) and quiescent Bmi1 ++4 position slow cycling stem cells ${ }^{59,60}$. Bmil + cells have been suggested as pre-terminal enteroendocrine cells ${ }^{61}$. Intestinal stem cells in each pool are highly heterogeneous. In addition, other slow cycling stem cells have been identified to express other ISC markers such as Lrig $1^{62}, H_{o p} x^{63}$, mTert $^{64}$. The identities and relationship of each type of stem cells with distinct ISC markers are not completely clear even after single-cell RNA sequencing of some progenitor pools that has defined hierarchical classification of some subpopulations ${ }^{46}$. Further studies to express Neurod 1 in mouse intestine using different progenitor and stem cell marker specific Cre drivers such as Atoh $1^{65}, \operatorname{Lgr} 5^{66}, B m i 1^{60}$ and $\operatorname{Lrig} 1^{62}$ may shed more light on the identities of those Neurod 1 susceptible early progenitor cells.

Similar to myogenic determining factor $M y o D$ that trans-differentiate fibroblasts into functional skeletal muscle cells ${ }^{67-69}$, Neurod 1 is able to direct neuron conversion from human cultured fibroblasts ${ }^{23}$ or in vivo endogenous mouse astrocytes in the brain and spinal cord ${ }^{70,71}$ when exogenously expressed. The interaction of Neurod 1 with Atoh1 plays a role in neurosensory cell differentiation during inner ear development and regeneration ${ }^{37,72,73}$. The potential of Neurod 1 to induce differentiation - including fate switching - in immature cells, suggests that it could play a major role in direct differentiation of cells, short-circuiting the current attempts to generate differentiated cells out of induced pluripotent stem cells. Consistent with this idea, recent studies showed that Neurod 1 can function as a pioneer factor and its expression in microglia can directly convert microglia to functional 
A.
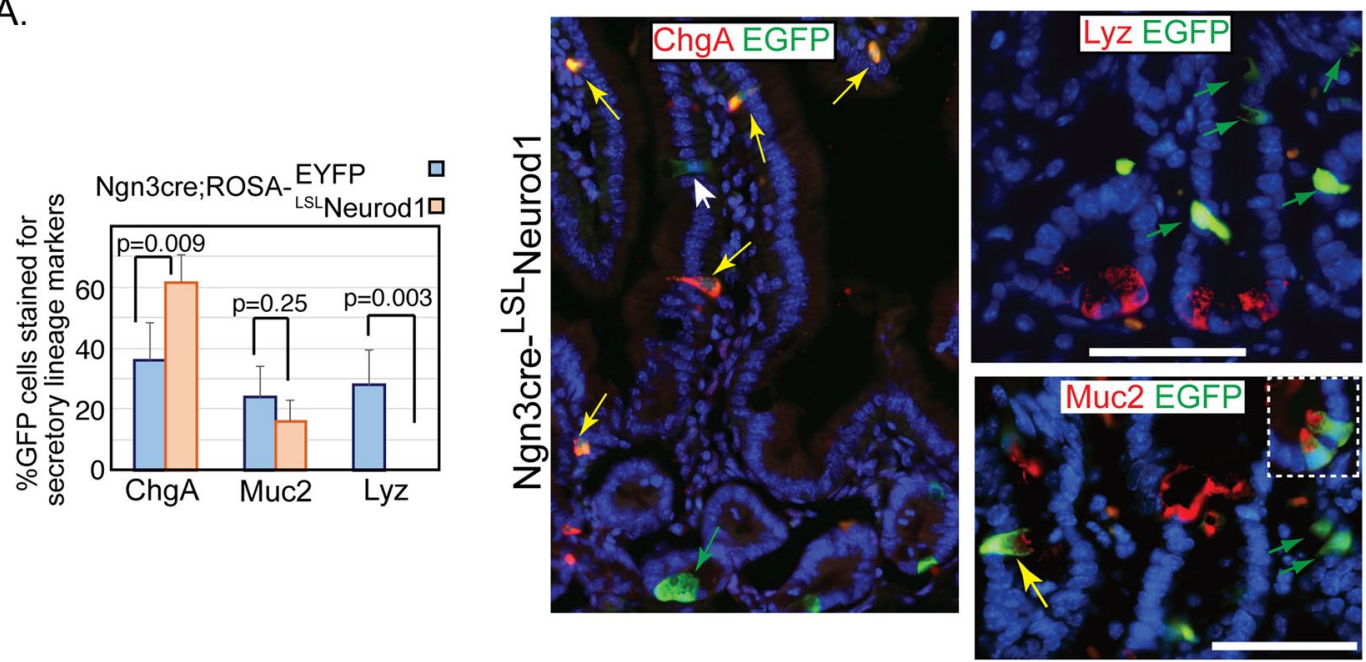

B.
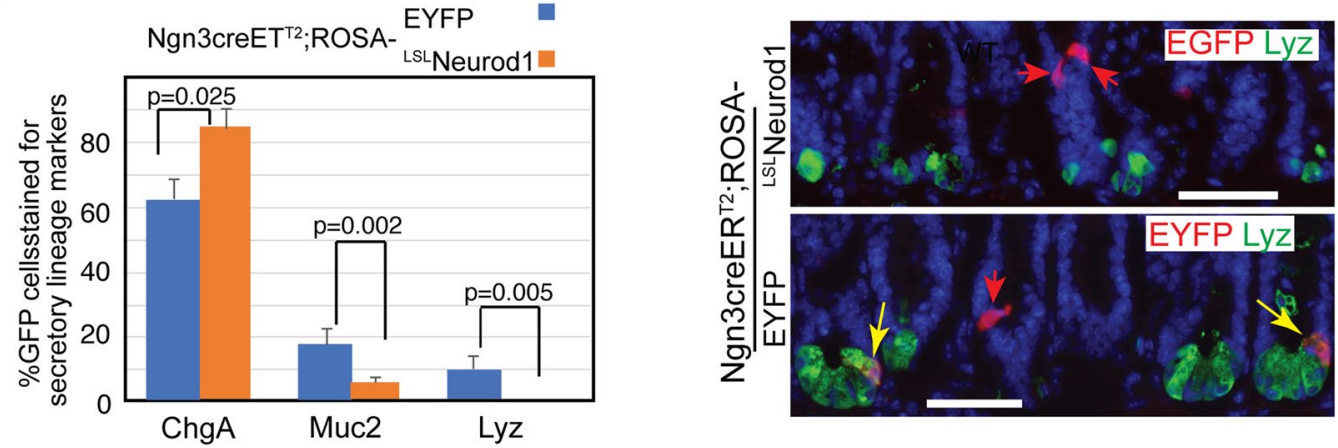

Figure 5. Ectopic expression of Neurod 1 in Neurog3 + cells in the intestine prevents Neurog $3+$ progenitor cells from becoming Paneth cells. Co-Immunofluorescent staining detected EGFP and the Paneth cell marker, lysozyme (Lyz), or goblet cell marker (Muc2) in the duodenum tissues of either Neurog3-cre- ${ }^{\text {LSL Neurod1 }}$ (A) or Tamoxifen-inducible Neurog3-creER ${ }^{\mathrm{T} 2}$; LSL Neurod1. (B) Scale bars represent $50 \mu \mathrm{m}$. Bar graphs on the left summarized the \% GFP+ intestinal epithelial cells stained for EEC (ChgA), Goblet (Muc2) and Paneth (Lyz) cells. Error bars represent standard deviation from at least three experimental replicates. P values were indicated in the graph.

neurons in the adult striatum ${ }^{47}$. Microglial cells are extremely plastic; it can undergo a variety of structure changes upon different stimuli. Like other pioneer factors, Neurod1 induces global transcriptional changes when exogenously expressed in microglia by occupying chromatin loci to modulate chromatin structure. Our preliminary results using a ChIP-seq assay identified over 7,500 substantial Neurod1 occupied loci in STC neuroendocrine cells (https://doi.org/10.1016/S0016-5085(17)30723-0), consistent with recent publication showing that nearly 3000 loci were regulated by Neurod $1^{74}$. Taken together, our data suggest a broader role of Neurod 1 as a possible pioneer factor in EEC differentiation.

We previously showed by lineage tracing using Neurog3-cre;ROSA-EYFP mice that Neurog3+ cells gave rise to not only EEC, but also Goblet and Paneth cells $s^{57}$. Here we found that the gain-of-function of Neurod1 expression in Neurog3 + cells blocked Neurog3 + cells from differentiating into Paneth cells (Fig. 5). Similarly, when Neurod1 was expressed in Villin + cells in Vilcre, ${ }^{\mathrm{LSL}}$ Neurod1 mice, we observed a considerable decrease in Paneth cell marker expression and no effect to Goblet and Tuft cell markers (Fig. 3). These results suggest that Neurod1 expression restricts intestinal epithelial cells towards EECs inhibiting Paneth cell lineage.

The canonical Wnt signaling pathway plays an important role not only in stem cell maintenance but also in Paneth cell differentiation and maturation processes ${ }^{75}$. Conditional expression of non-degradable mutant beta-catenin (exon3 deletion) in Neurod1 +EECs failed to activate wnt pathway, while the wnt activation occurred when the same mutant beta-catenin was expressed in Neurog $3+$ cells $^{76}$. The onset of Neurod1 expression resulted in the failure of Wnt activation and inhibition of its target gene c-Myc expression ${ }^{76}$. In fact, a number of tissue specific transcription factors have been described to interact with $\beta$-catenin and inhibit Wnt activation in a number of developmental systems including chondrogenesis and hematopoiesis ${ }^{77-83}$. Those interactions lead to blocking of differentiation through disrupting $\beta$-catenin/TCF4 complex (Icat, Hifla, Cdx2) or its binding to DNA (Runx3, Pdx1, Hbp1) or preventing coactivators recruitment (Klf4). Neurod1 might adopt similar mechanism in inhibiting Wnt activation. This may represent a novel yet unappreciated role of Neurod1 in mediating transcriptional repression of target gene expression. 


\section{Methods}

Mice. The Institutional Animal Care and Use Committee (IACUC) at the University of Massachusetts Medical School approved all experimental protocols of our vertebrate animal studies in accordance with National Institute of Health (NIH) guidelines. All mice were housed in AALACC certified facilities, under constant temperature, air pressure and specific pathogen-free conditions and a 12-hour light cycle.

For lineage tracing experiments, we used previously described Cre-driver mouse lines including Vil-cre ${ }^{49}$, Neurog3-cre ${ }^{57}$, Neurog3-cre ${ }^{\text {ERT2 }}$ and Neurod1-cre ${ }^{84}$. To induce Cre activity in Neurog 3 cre ${ }^{\text {ERT2 }}$ compounded mice, 4-week old mice were treated with $2 \mathrm{mg}$ tamoxifen (Sigma, T5648) dissolved in corn oil with one intraperitoneal injection daily for 5 consecutive days. Tissues were harvested and analyzed 7 days later.

Targeting loxp-neurod1-loxp ES cells. A pEntry-mNeurod1 plasmid was generated by cloning full-length mouse Neurod 1 cDNA into the pEntry vector (Gateway). A Gateway-compatible pROSA26 Destination Vector (pROSA26-DV1 LMBP 6350) ${ }^{48}$ was used to generate a Rosa26 locus targeting vector for conditional overexpression of Neurod 1 with an ires-EGFP reporter. Clones were verified by both enzymatic digestion and sequencing. A linearized ROSA26-DV1-mNeurod1 plasmid was electroporated into C57BL/6 derived JM8.F6 ES cells ${ }^{85}$. G418-resistant ES-cell clones were screened by PCR using primers set at both $5^{\prime}$ ROSA26 and $3^{\prime}$ covering loxP site: G1: 5'-TAGGTAGGGGATCGGGACTCT-3'; and G2: 5'-GCGAAGAGTTTGTCCTCAACC-3' to generate a $1.3 \mathrm{~kb}$ fragment. PCR-positive clones were further verified with additional PCR reactions using both Neurod1 internal and external ROSA-3'UTR primers and EGFP primers as indicated in Fig. 1: S1: ATCATGTCTGGATCCCCATC; S2: GGGGCGGAATTCGATATCAAG. mND552rev: TGGTAGTGGGCTGGGACAAACCTTT. Rosa3'UTR-DV1-for: AACAGAGGCTGTTGGTACTAGTGGC; Rosa3'UTRrev: AGCACCAAATGTGGTGCAGT. EGFP-for: AAG TTC ATC TGC ACC ACC G; EGFPrev: TCC TTG AAG AAG ATG GTG CG.

Generation of chimeras. The chimeras were generated by our Transgenic Core facility (https://www.umassmed. edu/tkomouse/). In brief, obtained positive JM8.F6 ES cell clones were injected into albino C57BL/6J $\mathrm{J}_{\mathrm{tyr}-\mathrm{Brd}}$ blastocysts. The resulting chimeras were bred with Albino C57BL/6 $\mathrm{J}_{\mathrm{tyr}-\mathrm{Brd}}$ mice. Germline transmitted transgenic mice that carried targeted allele were selected by fur color and confirmed by genotyping using PCR analysis of DNA isolated from mice ear snips. Primer pairs S1:mND552rev shown in Fig. 1 were used in the PCR reaction.

Intestinal epithelial cell isolation. Mouse intestinal crypt and villus isolation were performed according to a published protocol with modifications ${ }^{86}$. In brief, mice were euthanized by $\mathrm{CO}_{2}$ and cervical dislocation. The small intestines were flushed with ice-cold $1 \mathrm{xPBS}$ and everted on a $4 \mathrm{~mm}$ rod. Intestinal tissues were cut into $2 \mathrm{~cm}$ pieces and incubated in chelating buffer $\left[\mathrm{pH}=7.3\right.$ : 1xD-PBS $\left(1 \mathrm{mM} \mathrm{CaC}_{\mathrm{l} 2}, 0.5 \mathrm{mM} \mathrm{MgC}_{\mathrm{l} 2}, 8 \mathrm{mM} \mathrm{Na}_{2} \mathrm{HPO}_{4}, 137\right.$ mM-NaCl, $\left.1.5 \mathrm{mM}-\mathrm{KH}_{2} 2 \mathrm{PO}_{4}, 2.7 \mathrm{mM}-\mathrm{KCl}\right), 1 \mathrm{mM}-\mathrm{DTT}, 1 \mathrm{mM}$ EDTA] at $4{ }^{\circ} \mathrm{C}$ with constant shaking for $30 \mathrm{~min}$. The cells were released as large sheets of villus cells. The tissues were transferred to fresh chelating buffer and were vigorously shaken to release crypts into the media. The collected villus and crypt portions were filtered through a $70 \mu \mathrm{m}$ Cell Strainer and pelleted at $200 \mathrm{rpm}$ for 5 minutes with low brake speed. The villus and crypt pellets were resuspended in Trizol (Invitrogen, Inc.) for RNA extraction.

RNA isolation and qPCR analysis. RNAs were prepared with RNeasy Mini kit (Qiagen). In-column DNA digestion was performed to remove possible DNA contamination using RNase-free DNase (Qiagen). cDNAs were prepared using the Maxima First-Strand cDNA synthesis kit for RT-qPCR (Thermo Scientific). qPCRs were performed according to standard protocol using the Light Cycler (BioRad). Results are given as relative expression normalized to housekeeping gene beta-actin. Primer sequences are available upon request.

Immunofluorescence staining and western blotting. Intestinal tissues were fixed in $4 \%$ formaldehyde and embedded in OCT for frozen sections. For immunofluorescence analysis, the following primary antibodies were used: goat anti-EGFP (1:400, Novus Biologicals: NB100-1770), rabbit anti-Chromogranin A (1:1000, Immunostar: \#20085), rabbit anti-Muc2 (1:500, Santa Cruz: H-300), and rabbit anti-Lyz (1:500, Zymed Laboratories: 18-0039), rabbit anti-Neurod1 (1:250, Abcam: Ab109224). Alexa Fluor 488 or 594 conjugated secondary antibodies were used at a dilution of 1:800. Intestinal Alkaline Phosphatase activities were detected according to the manufacturer's protocol (Vector Red AP Substrate Kit, Cat. \#: SK-5100). Images were aquired using a Nikon Eclipse E600 microscope with imaging software of NIS-Elements AR 4.30.02.

Western blotting was performed as previous describe ${ }^{22}$. In brief, equal amount of proteins was separated by SDS-PAGE and eleterotransferred onto a nitrocellulose membrane. Nonspecific binding to the membrane was blocked with a $3 \%$ milk solution in phosphate-buffered saline (PBS) containing $0.1 \%$ NP-40 prior to incubation with an anti-Neurod1 antibody (1:1,000; Abcam clone \#Ab109224) or anti-actin antibody (1:2,000, Santa Cruz: sc-1616 HRP). An enhanced chemiluminescence (ECL) kit (Amersham) was used for detection of protein.

Statistical analysis. Pair-wise comparisons were analyzed by the Student's $t$ test. The statistical significance of pairwise comparisons shown on the bar graph were indicated with $P \leq 0.05$ as significant and $\mathrm{P}>0.05$ as non-significant.

\section{Data availability}

No datasets were generated or analyzed during the current study.

Received: 6 August 2019; Accepted: 25 November 2019;

Published online: 20 December 2019 


\section{References}

1. de Santa Barbara, P., van den Brink, G. R. \& Roberts, D. J. Development and differentiation of the intestinal epithelium. Cellular and molecular life sciences: CMLS 60, 1322-1332 (2003).

2. Noah, T. K., Donahue, B. \& Shroyer, N. F. Intestinal development and differentiation. Experimental cell research 317, 2702-2710 (2011).

3. Yang, Q., Bermingham, N. A., Finegold, M. J. \& Zoghbi, H. Y. Requirement of Math1 for secretory cell lineage commitment in the mouse intestine. Science 294, 2155-2158 (2001).

4. Shroyer, N. F., Wallis, D., Venken, K. J., Bellen, H. J. \& Zoghbi, H. Y. Gfil functions downstream of Math1 to control intestinal secretory cell subtype allocation and differentiation. Genes \& development 19, 2412-2417 (2005).

5. Noah, T. K., Kazanjian, A., Whitsett, J. \& Shroyer, N. F. SAM pointed domain ETS factor (SPDEF) regulates terminal differentiation and maturation of intestinal goblet cells. Experimental cell research 316, 452-465 (2010).

6. Lopez-Diaz, L. et al. Intestinal Neurogenin 3 directs differentiation of a bipotential secretory progenitor to endocrine cell rather than goblet cell fate. Developmental biology 309, 298-305 (2007).

7. Li, H. J., Ray, S. K., Singh, N. K., Johnston, B. \& Leiter, A. B. Basic helix-loop-helix transcription factors and enteroendocrine cell differentiation. Diabetes Obes Metab 13(Suppl 1), 5-12 (2011).

8. Gracz, A. D. et al. Sox4 Promotes Atoh1-Independent Intestinal Secretory Differentiation Toward Tuft and Enteroendocrine Fates. Gastroenterology 155, 1508-1523 e1510, https://doi.org/10.1053/j.gastro.2018.07.023 (2018).

9. Gerbe, F. et al. Distinct ATOH1 and Neurog3 requirements define tuft cells as a new secretory cell type in the intestinal epithelium. JCell Biol 192, 767-780 (2011).

10. Mutoh, H. et al. The basic helix-loop-helix transcription factor BETA2/NeuroD is expressed in mammalian enteroendocrine cells and activates secretin gene expression. Proceedings of the National Academy of Sciences of the United States of America 94, 3560-3564 (1997).

11. Naya, F. J. et al. Diabetes, defective pancreatic morphogenesis, and abnormal enteroendocrine differentiation in BETA2/neuroDdeficient mice. Genes \& development 11, 2323-2334 (1997).

12. Gross, S. et al. The novel enterochromaffin marker Lmxla regulates serotonin biosynthesis in enteroendocrine cell lineages downstream of Nkx2.2. Development 143, 2616-2628 (2016).

13. Desai, S. et al. Nkx2.2 regulates cell fate choice in the enteroendocrine cell lineages of the intestine. Developmental biology 313 , 58-66 (2008).

14. Wang, Y. C. et al. Homeodomain transcription factor NKX2.2 functions in immature cells to control enteroendocrine differentiation and is expressed in gastrointestinal neuroendocrine tumors. Endocrine-related cancer 16, 267-279 (2009).

15. Gierl, M. S., Karoulias, N., Wende, H., Strehle, M. \& Birchmeier, C. The zinc-finger factor Insm1 (IA-1) is essential for the development of pancreatic beta cells and intestinal endocrine cells. Genes \& development 20, 2465-2478 (2006).

16. Du, A. et al. Arx is required for normal enteroendocrine cell development in mice and humans. Developmental biology 365, 175-188 (2012).

17. Beucher, A. et al. The homeodomain-containing transcription factors Arx and Pax 4 control enteroendocrine subtype specification in mice. PloS one 7, e36449 (2012).

18. Terry, N. A., Walp, E. R., Lee, R. A., Kaestner, K. H. \& May, C. L. Impaired enteroendocrine development in intestinal-specific Islet1 mouse mutants causes impaired glucose homeostasis. American journal of physiology. Gastrointestinal and liver physiology 307, G979-991 (2014).

19. Hill, M. E., Asa, S. L. \& Drucker, D. J. Essential requirement for Pax6 in control of enteroendocrine proglucagon gene transcription. Mol Endocrinol 13, 1474-1486 (1999).

20. Naya, F. J., Stellrecht, C. M. \& Tsai, M. J. Tissue-specific regulation of the insulin gene by a novel basic helix-loop-helix transcription factor. Genes \& development 9, 1009-1019 (1995).

21. Gu, C. et al. Pancreatic beta cells require NeuroD to achieve and maintain functional maturity. Cell Metab 11, 298-310 (2010)

22. Ray, S. K., Nishitani, J., Petry, M. W., Fessing, M. Y. \& Leiter, A. B. Novel transcriptional potentiation of BETA2/NeuroD on the secretin gene promoter by the DNA-binding protein Finb/RREB-1. Molecular and cellular biology 23, 259-271 (2003).

23. Pang, Z. P. et al. Induction of human neuronal cells by defined transcription factors. Nature 476, 220-223 (2011).

24. Wapinski, O. L. et al. Hierarchical mechanisms for direct reprogramming of fibroblasts to neurons. Cell 155, 621-635 (2013).

25. Vierbuchen, T. et al. Direct conversion of fibroblasts to functional neurons by defined factors. Nature 463, 1035 (2010).

26. Noda, T. et al. Direct Reprogramming of Spiral Ganglion Non-neuronal Cells into Neurons: Toward Ameliorating Sensorineural Hearing Loss by Gene Therapy. Frontiers in cell and developmental biology 6, 16 (2018).

27. Ochocinska, M. \& Hitchcock, P. NeuroD regulates proliferation of photoreceptor progenitors in the retina of the zebrafish. Mechanisms of development 126, 128-141 (2009).

28. Mutoh, H., Naya, F. J., Tsai, M. J. \& Leiter, A. B. The basic helix-loop-helix protein BETA2 interacts with p300 to coordinate differentiation of secretin-expressing enteroendocrine cells. Genes \& development 12, 820-830 (1998).

29. Farah, M. H. et al. Generation of neurons by transient expression of neural bHLH proteins in mammalian cells. Development 127, 693-702 (2000).

30. Hevner, R. F., Hodge, R. D., Daza, R. A. \& Englund, C. Transcription factors in glutamatergic neurogenesis: conserved programs in neocortex, cerebellum, and adult hippocampus. Neuroscience research 55, 223-233 (2006).

31. Miyata, T., Maeda, T. \& Lee, J. E. NeuroD is required for differentiation of the granule cells in the cerebellum and hippocampus. Genes \& development 13, 1647-1652 (1999).

32. Liu, M. et al. Loss of BETA2/NeuroD leads to malformation of the dentate gyrus and epilepsy. Proceedings of the National Academy of Sciences of the United States of America 97, 865-870 (2000).

33. Schwab, M. H. et al. Neuronal basic helix-loop-helix proteins (NEX and BETA2/Neuro D) regulate terminal granule cell differentiation in the hippocampus. J Neurosci 20, 3714-3724 (2000).

34. Kim, W. Y. et al. NeuroD-null mice are deaf due to a severe loss of the inner ear sensory neurons during development. Development 128, 417-426 (2001).

35. Jahan, I., Pan, N., Kersigo, J. \& Fritzsch, B. Neurod1 suppresses hair cell differentiation in ear ganglia and regulates hair cell subtype development in the cochlea. PLoS One 5, e11661 (2010).

36. Liu, M. et al. Essential role of BETA2/NeuroD1 in development of the vestibular and auditory systems. Genes \& development 14, 2839-2854 (2000).

37. Macova, I. et al. Neurod1 is essential for the primary tonotopic organization and related auditory information processing in the midbrain. Journal of Neuroscience 39, 984-1004 (2019).

38. Rindi, G. et al. Targeted ablation of secretin-producing cells in transgenic mice reveals a common differentiation pathway with multiple enteroendocrine cell lineages in the small intestine. Development 126, 4149-4156 (1999).

39. Li, H. J. et al. Distinct cellular origins for serotonin-expressing and enterochromaffin-like cells in the gastric corpus. Gastroenterology 146, 754-764 e753 (2014).

40. Itkin-Ansari, P. et al. NeuroD1 in the endocrine pancreas: localization and dual function as an activator and repressor. Developmental dynamics: an official publication of the American Association of Anatomists 233, 946-953 (2005).

41. Malecki, M. T. et al. Mutations in NEUROD1 are associated with the development of type 2 diabetes mellitus. Nature genetics 23, 323-328 (1999). 
42. Orosz, O. et al. Ophthalmological phenotype associated with homozygous null mutation in the NEUROD1 gene. Mol Vis 21, 124-130 (2015)

43. Anderson, K. R. et al. Cooperative transcriptional regulation of the essential pancreatic islet gene NeuroD1 (beta2) by Nkx2.2 and neurogenin 3. The Journal of biological chemistry 284, 31236-31248 (2009).

44. Huang, H. P. et al. Regulation of the pancreatic islet-specific gene BETA2 (neuroD) by neurogenin 3. Molecular and cellular biology 20, 3292-3307 (2000).

45. Haber, A. L. et al. A single-cell survey of the small intestinal epithelium. Nature 551, 333-339 (2017).

46. Gehart, H. et al. Identification of Enteroendocrine Regulators by Real-Time Single-Cell Differentiation Mapping. Cell 176, 1158-1173 e1116 (2019).

47. Matsuda, T. et al. Pioneer Factor NeuroD1 Rearranges Transcriptional and Epigenetic Profiles to Execute Microglia-Neuron Conversion. Neuron 101, 472-485 e477 (2019).

48. Nyabi, O. et al. Efficient mouse transgenesis using Gateway-compatible ROSA26 locus targeting vectors and F1 hybrid ES cells. Nucleic acids research 37, e55 (2009).

49. el Marjou, F. et al. Tissue-specific and inducible Cre-mediated recombination in the gut epithelium. Genesis 39, 186-193 (2004).

50. Madison, B. B. et al. Cis elements of the villin gene control expression in restricted domains of the vertical (crypt) and horizontal (duodenum, cecum) axes of the intestine. The Journal of biological chemistry 277, 33275-33283 (2002).

51. Houdebine, L. M. \& Attal, J. Internal ribosome entry sites (IRESs): reality and use. Transgenic research 8, 157-177 (1999).

52. Taboit-Dameron, F. et al. Association of the $5^{\prime} \mathrm{HS} 4$ sequence of the chicken beta-globin locus control region with human EF1 alpha gene promoter induces ubiquitous and high expression of human CD55 and CD59 cDNAs in transgenic rabbits. Transgenic research 8, 223-235 (1999)

53. Deftos, L. J. Chromogranin A: its role in endocrine function and as an endocrine and neuroendocrine tumor marker. Endocr Rev 12, 181-187 (1991)

54. Jensen, J. et al. Control of endodermal endocrine development by Hes-1. Nature genetics 24, 36-44 (2000).

55. Pan, N., Jahan, I., Lee, J. E. \& Fritzsch, B. Defects in the cerebella of conditional Neurod1 null mice correlate with effective Tg (Atoh1-cre) recombination and granule cell requirements for Neurod1 for differentiation. Cell and tissue research 337, 407-428 (2009).

56. Jahan, I., Pan, N., Elliott, K. L. \& Fritzsch, B. The quest for restoring hearing: understanding ear development more completely. Bioessays 37, 1016-1027 (2015).

57. Schonhoff, S. E., Giel-Moloney, M. \& Leiter, A. B. Neurogenin 3-expressing progenitor cells in the gastrointestinal tract differentiate into both endocrine and non-endocrine cell types. Developmental biology 270, 443-454 (2004).

58. Liu, J. et al. Non-parallel recombination limits Cre-LoxP-based reporters as precise indicators of conditional genetic manipulation. Genesis 51, 436-442 (2013).

59. Li, L. \& Clevers, H. Coexistence of quiescent and active adult stem cells in mammals. Science 327, 542-545 (2010).

60. Sangiorgi, E. \& Capecchi, M. R. Bmil is expressed in vivo in intestinal stem cells. Nature genetics 40, 915-920 (2008).

61. Jadhav, U. et al. Dynamic Reorganization of Chromatin Accessibility Signatures during Dedifferentiation of Secretory Precursors into Lgr5+ Intestinal Stem Cells. Cell stem cell 21, 65-77 e65 (2017).

62. Ordonez-Moran, P. \& Huelsken, J. Lrig1: a new master regulator of epithelial stem cells. The EMBO journal 31, 2064-2066 (2012).

63. Takeda, N. et al. Interconversion between intestinal stem cell populations in distinct niches. Science 334, 1420-1424 (2011).

64. Breault, D. T. et al. Generation of mTert-GFP mice as a model to identify and study tissue progenitor cells. Proceedings of the National Academy of Sciences of the United States of America 105, 10420-10425 (2008).

65. VanDussen, K. L. \& Samuelson, L. C. Mouse atonal homolog 1 directs intestinal progenitors to secretory cell rather than absorptive cell fate. Developmental biology 346, 215-223 (2010).

66. Barker, N. \& Clevers, H. Leucine-rich repeat-containing G-protein-coupled receptors as markers of adult stem cells. Gastroenterology 138, 1681-1696 (2010)

67. Weintraub, H. et al. Activation of muscle-specific genes in pigment, nerve, fat, liver, and fibroblast cell lines by forced expression of MyoD. Proceedings of the National Academy of Sciences 86, 5434-5438 (1989).

68. Lahmann, I. et al. Oscillations of MyoD and Hesl proteins regulate the maintenance of activated muscle stem cells. Genes \& development (2019)

69. Boularaoui, S. M. et al. Efficient transdifferentiation of human dermal fibroblasts into skeletal muscle. Journal of tissue engineering and regenerative medicine 12, e918-e936 (2018).

70. Brulet, R., Zhu, J., Aktar, M., Hsieh, J. \& Cho, K. O. Mice with conditional NeuroD1 knockout display reduced aberrant hippocampal neurogenesis but no change in epileptic seizures. Exp Neurol 293, 190-198 (2017)

71. Su, Z., Niu, W., Liu, M. L., Zou, Y. \& Zhang, C. L. In vivo conversion of astrocytes to neurons in the injured adult spinal cord. Nature communications 5, 3338 (2014).

72. Pan, N., Jahan, I., Lee, J. E. \& Fritzsch, B. Defects in the cerebella of conditional Neurod1 null mice correlate with effective Tg(Atoh1cre) recombination and granule cell requirements for Neurod1 for differentiation. Cell Tissue Res 337, 407-428 (2009).

73. Jahan, I., Elliott, K. L. \& Fritzsch, B. Understanding Molecular Evolution and Development of the Organ of Corti Can Provide Clues for Hearing Restoration. Integr Comp Biol 58, 351-365 (2018).

74. Pataskar, A. et al. NeuroD1 reprograms chromatin and transcription factor landscapes to induce the neuronal program. The EMBO journal 35, 24-45 (2016).

75. Gregorieff, A. \& Clevers, H. Wnt signaling in the intestinal epithelium: from endoderm to cancer. Genes \& development 19, 877-890 (2005).

76. Wang, Y., Giel-Moloney, M., Rindi, G. \& Leiter, A. B. Enteroendocrine precursors differentiate independently of Wnt and form serotonin expressing adenomas in response to active beta-catenin. Proceedings of the National Academy of Sciences of the United States of America 104, 11328-11333 (2007).

77. Evans, P. M., Chen, X., Zhang, W. \& Liu, C. KLF4 interacts with beta-catenin/TCF4 and blocks p300/CBP recruitment by betacatenin. Molecular and cellular biology 30, 372-381 (2010).

78. Ito, K. et al. RUNX3 attenuates beta-catenin/T cell factors in intestinal tumorigenesis. Cancer Cell 14, 226-237 (2008).

79. Chen, L. et al. Negative regulation of c-Myc transcription by pancreas duodenum homeobox-1. Endocrinology 148, 2168-2180 (2007).

80. Guo, R. J., Funakoshi, S., Lee, H. H., Kong, J. \& Lynch, J. P. The intestine-specific transcription factor Cdx2 inhibits beta-catenin/TCF transcriptional activity by disrupting the beta-catenin-TCF protein complex. Carcinogenesis 31, 159-166

81. Lim, J. H., Chun, Y. S. \& Park, J. W. Hypoxia-inducible factor-1alpha obstructs a Wnt signaling pathway by inhibiting the hARD1mediated activation of beta-catenin. Cancer Res 68, 5177-5184 (2008).

82. Sampson, E. M. et al. Negative regulation of the Wnt-beta-catenin pathway by the transcriptional repressor HBP1. The EMBO journal 20, 4500-4511, https://doi.org/10.1093/emboj/20.16.4500 (2001).

83. Zhang, K. et al. ICAT inhibits glioblastoma cell proliferation by suppressing Wnt/beta-catenin activity. Cancer Lett 357, 404-411 (2015).

84. Li, H. J., Kapoor, A., Giel-Moloney, M., Rindi, G. \& Leiter, A. B. Notch signaling differentially regulates the cell fate of early endocrine precursor cells and their maturing descendants in the mouse pancreas and intestine. Developmental biology 371, 156-169 (2012).

85. Pettitt, S. J. et al. Agouti C57BL/6N embryonic stem cells for mouse genetic resources. Nature methods 6, 493-495 (2009).

86. Flint, N., Cove, F. L. \& Evans, G. S. A low-temperature method for the isolation of small-intestinal epithelium along the crypt-villus axis. Biochem J 280(Pt 2), 331-334 (1991). 


\section{Acknowledgements}

The authors thank the transgenic core facility at UMASS Med school and Dr. Junhao Mao and Mrs. Melanie Trombly for critical reading and manuscript editing. This work was in part supported by NIH (R01 DK100223 and R01 DK110614 to AR; R01 AG060504 to BF).

\section{Author contributions}

H.J.L. and A.B.L. conceived the project, H.J.L. generated the transgenic mice, H.J.L. and S.R. performed the experiments and collected the data, J.H. and N.P. provided plasmids for generating conditional mNeurod 1 targeting vector. H.J.L., A.L. and B.F. wrote the paper.

\section{Competing interests}

The authors declare no competing interests.

\section{Additional information}

Supplementary information is available for this paper at https://doi.org/10.1038/s41598-019-55292-7.

Correspondence and requests for materials should be addressed to A.B.L.

Reprints and permissions information is available at www.nature.com/reprints.

Publisher's note Springer Nature remains neutral with regard to jurisdictional claims in published maps and institutional affiliations.

(c) (i) Open Access This article is licensed under a Creative Commons Attribution 4.0 International License, which permits use, sharing, adaptation, distribution and reproduction in any medium or format, as long as you give appropriate credit to the original author(s) and the source, provide a link to the Creative Commons license, and indicate if changes were made. The images or other third party material in this article are included in the article's Creative Commons license, unless indicated otherwise in a credit line to the material. If material is not included in the article's Creative Commons license and your intended use is not permitted by statutory regulation or exceeds the permitted use, you will need to obtain permission directly from the copyright holder. To view a copy of this license, visit http://creativecommons.org/licenses/by/4.0/.

(c) The Author(s) 2019 